\title{
An Adaptive Mesh Smoothing Method Based on a Probabilistic Error Indicator
}

\author{
S. H. Dibajian ${ }^{1 *}$, S. H. Hashemolhoseini' ${ }^{2}$ M. Farzin' and M. Gandomkar ${ }^{1}$ \\ 'Department of Mechanical Engineering, Isfahan University of Technology, Isfahan, Iran; \\ dibajian@me.iut.ac.ir, hamidh@cc.iut.ac.ir, Farzin@cc.iut.ac.ir,mgd_gandomkar@me.iut.ac.ir \\ 2Department of Mineral Engineering, Isfahan University of Technology, Isfahan, Iran; hamidh@cc.iut.ac.ir
}

\begin{abstract}
In this paper assessment of the reliability of the results in the finite element method is studied by using probability theory. A probabilistic error indicator is presented which is based on Kriging error interpolation and is used for mesh smoothing purpose. Effective parameters such as shape functions, volume of elements and distance between nodes are considered simultaneously. The field variation is also considered and is calculated statistically or by other means such as user's sense. This error indicator is used for adaptive mesh smoothing purposes especially in the uncoupled ALE approaches. Robustness and accuracy of the method are shown through several examples on different fields with irregular boundaries or abrupt changes.
\end{abstract}

Keywords: Adaptive Mesh, Error Estimator, Finite Element, Mesh Smoothing

\section{Introduction}

In the FEM analyses, mesh quality affects solution accuracy. Hence this quality should be improved via smoothing. Mesh smoothing algorithms relocate the nodes of the previous mesh to improve mesh quality. The field variation is also an important factor which affects the mesh quality. Although field variation has been used in some mesh refinement applications such as ALE approaches, generally it is not considered in the conventional smoothing methods.

In literature, the mesh quality is often characterized by the smoothness or shape of the elements. Some measures such as aspect ratio, change in elemental area and the angles subtended at a node are commonly used to quantify the smoothness of a mesh. One popular mesh smoothing algorithm is the Laplacian smoothing which relocates a node by calculating the average of the adjacent nodes coordinates which are connected by element edges to the node in question ${ }^{1}$. Volume smoothing relocates a node by computing a volume-weighted average of the element centers in the elements surrounding the node.
This approach can be considered as a modified Laplacian smoothing. Similarly, there are various mesh smoothing algorithms which are based on distances between points ${ }^{2-8}$. A number of other mesh smoothing methods, produce acceptable quality meshes by minimizing a distortion metric ${ }^{9,10}$. Also some approaches are angle-based optimization ${ }^{11,12}$. In order to generate better results, some researchers combine two or more smoothing methods $s^{13,14}$. However no error estimation is performed in the above mentioned smoothing methods.

To the author's opinion error estimation, evaluated based on variation of field variable can be a logical basis for improvement of mesh quality. Although there are some error estimators in literature ${ }^{15-23}$, these methods have not been directly used to improve mesh quality and mesh refinement. In these methods only field variation has been considered for error indication. In these formulations there is not any measure to show the effect of relocation of nodes to reduce errors. These methods should be used in successive runs and they do not provide any exact clue about size of required elements. 
Hence, available techniques are either mesh refinement based on error estimation of the field which considers field variable or are smoothing methods such as Laplacian or volumetric method etc. In this study, an adaptive mesh smoothing method is presented which eliminates the above mentioned shortcomings. To the authors' knowledge there is no available adaptive mesh smoothing method which directly performs a mesh smoothing as well as mesh refinement based on field variation and its error estimation. An error indicator is presented and then is used directly to improve mesh quality. In this method, the degree of belief on the accuracy of discretization which is evaluated by a probabilistic method is used as an error indicator. This error indicator considers parameters such as shape functions, volume of the elements, distance between nodes and field variation simultaneously. Therefore this indicator can be used in $\mathrm{r}, \mathrm{h}$ or $\mathrm{p}$ refinement methods. However in this paper, the presented error indicator is only used for $\mathrm{r}$ refinement mesh smoothing.

A new aspect of this paper is its probabilistic methodology which is also implemented for the first time. The fundamentals of the probabilistic approach used in this study are based on Kriging interpolation method ${ }^{24}$. Assumptions used in traditional Kriging method have been modified to be applicable for numerical analyses used in this paper.

This adaptive mesh smoothing automatically yields an optimized mesh structure from a smooth to a coarse mesh as a function of field variation and its error. In available mesh refinement techniques in literature, error of the filed variable is estimated then mesh refinement is performed but region of refinement is not well defined and this refinement should be performed in several runs. While in the present method region of refinement is automatically obtained. Even the sizes of refined elements are obtained based on acceptable error values. Using this method number of elements are kept constant and sizes of the elements are automatically altered. Hence it would be beneficial in terms of computation time since number of nodes and elements are not increased.

Capability of this adaptive mesh smoothing to reconstruct various mesh geometries is examined by several simple examples such as smoothing near the boundaries and near a load concentration. Then capability of error estimator and stability of method is shown by a rectangular plate with a circular hole under in plain loading which is usually used as a bench mark in structural problems. Finally the application of this method in a three dimensional thermal analysis of flame forming process is also explained. This example is an illustration of the ability of the presented method in ALE refinement technique.

If heating path is simply a straight line, an appropriate mesh can be created by the user, nevertheless in complex heating paths it is difficult to obtain anadequately refined mesh near the region of interest. In these cases using adaptivity techniques help to obtain quality solutions while the cost of the analysis is reduced. The fundamentals of the probabilistic approach used in this study are explained in the next section.

\section{Interpretation of Probability}

Probability theory is an appropriate approach to handle uncertainty in mathematics. There are several categories of probability interpretations such as classical probability, frequency probability, subjective probability and objective probability. In the elementary probabilistic textbooks, classical definition of probability is used. Definition of classical probability is "ratio between the favorable cases and the number of equally probable cases". Since there are some paradoxes in this definition, frequency probability is more used in the engineering textbooks. This interpretation defines an event's probability as the limit of its relative frequency in a large number of trials. Therefore frequency probability is based on the idea that some identical events exist. Obviously it is not a perfect idea. In the other word repeated trials do not exist in reality.

On the contrary of the above mentioned interpretations, according to subjective probability, "Probability depends on the Subject's State of Information"25. Therefore if the state of knowledge is alike, the evaluation of probability would coincide and "for an omniscient being probability would not exist"25.

Now referring to the PDE's, usually there is no explicit solution to these equations. Therefore some researchers focus on finding approximate solution methods such as FEM. Since FEM solutions depend on the mesh and other assumptions used in the formulations, the field variable (denoted here by $f(x)$ ) is generally nondeterministic. In fact the state of information about exact solution affects the probability of the approximate solution and its accuracy. For someone who is aware of the exact solution the field variable and its accuracy are deterministic. Since the circumstances for field variable in finite element method 
and subjective probability are the same, therefore this paper will be constructed based on this probabilistic idea.

In the FEM, the solution precision is extremely affected by the interpolation procedure. For example if an element base function matches the exact field, the exact solution can be obtained by the FEM. Therefore if the degree of belief (or the degree of unbelief) in the behavior of interpolation of the field variable is measured, an error indicator will be obtained. Strictly speaking, in the present research, the degree of belief is used as an error estimator instead of traditional methods.

In common problems, variance is a suitable function that can be used to evaluate the degree of belief. For example for a given temperature state, if temperature is measured several times by one thermometer, the degree of belief in the precision of that thermometer can be evaluated by variance of these temperatures. In the present study similar approach is used to evaluate the degree of belief in the accuracy of interpolation. However there is only one solution at every point in the FEM. Therefore the variance function cannot be calculated directly.

Suppose FEM procedure is a black box and nodal values $\left(f_{i}\right)$ are its outputs. These outputs are considered as a realization of the field. Since these outputs are samples of a smooth field, the values of the field at every arbitrary point can be approximated by vicinal samples. In other words, there is a certain correlation between nearby samples. In this paper, this correlation between data at nearby spatial locations is used to calculate the variance and evaluate the degree of belief in the accuracy of interpolation used in FEM. This idea which was used by Matheron ${ }^{24}$ for the first time in the geostatistic applications will be rewritten in the next section in our FEM approach.

\section{Error Estimator and Mesh Smoothing}

As was mentioned in the previous section, the field variable $f(\mathrm{x})$ and the results of FE method $\left(f_{i}\right)$ are considered as nondeterministic values and realization of the field respectively. In the FE method, approximation of the field is an important concept which is obtained by the following assumption:

$$
f_{h}(\mathbf{x})=\sum_{i=1}^{n} \varphi_{i} f_{i}
$$

in which, $\varphi_{i}$ 's and $f_{j}$ 's are shape functions and nodal values respectively. In this paper, error indicator is defined as follows:

$$
\text { error indicator }=\sigma_{E}^{2}=E\left(\left(f(\mathbf{x})-f_{b}\right)^{2}\right)
$$

where, $f(\mathrm{x})$ is the field variable and $\mathrm{E}(f)$ the mathematical expectation of $f$. $\mathrm{E}(f)$ is a linear function which expresses expected value of $f$. Equation 2 can be expanded as follows:

$$
\begin{aligned}
\sigma_{E}^{2}(\mathbf{x})= & E\left(\left(f(\mathbf{x})-f_{b}(\mathbf{x})\right)^{2}\right)=E\left(f^{2}(\mathbf{x})\right)+\sum_{j=1}^{n} \sum_{i=1}^{n} \varphi_{i} \varphi_{j} E\left(f_{i} \cdot f_{j}\right) \\
& -2 \sum_{i=1}^{n} \varphi_{i} E\left(f_{i} \cdot f(\mathbf{x})\right)
\end{aligned}
$$

It should be noted that generally $\mathrm{E}(f)$ can be evaluated statistically by Arithmetic Mean. Since there are not sufficient data to evaluate this function, Eq. (3) must be rewritten as a function of appropriate parameters. Sincecovariance is defined as follows:

$$
\begin{aligned}
\operatorname{Cov}\left(f_{1}, f_{2}\right)= & E\left(\left(f_{1}-E\left(f_{1}\right)\right)\left(f_{2}-E\left(f_{2}\right)\right)\right)=E\left(f_{1} f_{2}\right)- \\
& E\left(f_{1}\right) E\left(f_{2}\right)
\end{aligned}
$$

Therefore:

$$
\begin{aligned}
\sigma_{E}^{2}(\mathbf{x})= & \operatorname{Cov}(f(\mathbf{x}), f(\mathbf{x}))+\sum_{j=1}^{n} \sum_{i=1}^{n} \varphi_{i} \varphi_{j} \operatorname{Cov}\left(f_{i}, f_{j}\right)- \\
& 2 \sum_{i=1}^{n} \varphi_{i} C\left(f_{i}, f(\mathbf{x})\right)
\end{aligned}
$$

It should be noted that in probabilistic theory, covariance function $\operatorname{Cov}\left(f_{i}, f_{j}\right)$ represents the correlation between $f_{i}$ and $f_{j}$ which are evaluated at points $x_{i}$ and $x_{j}$ respectively. Due to continuity of the field variable, in sufficiently small vicinity of two points, the difference between $f_{i}$ and $f_{j}$ will decrease if distance between $x_{i}$ and $x_{j}$ decreases. Disregarding other parameters, $\operatorname{Cov}\left(f_{i}, f_{j}\right)$ is a function of distance between $\mathbf{x}_{i}$ and $\mathbf{x}_{j}$ and can be represented as follows:

$$
\operatorname{Cov}\left(f_{i}, f_{j}\right)=C\left(\mathbf{x}_{i}, \mathbf{x}_{j}\right)=C\left(\left\|\mathbf{x}_{i}-\mathbf{x}_{j}\right\|\right)=C\left(h_{i j}\right)
$$

where, $\mathrm{C}$ is a certain function. Therefore equation 5 can be rewritten:

$$
\sigma_{E}^{2}(\mathbf{x})=C(0)+\sum_{j=1}^{n} \sum_{i=1}^{n} \varphi_{i} \varphi_{j} C\left(h_{i j}\right)-2 \sum_{i=1}^{n} \varphi_{i} C\left(h_{i}\right)
$$

where, $h_{i j}$ and $h_{i}$ are distances between $\mathbf{x}_{i}, \mathbf{x}_{j}$ and $x_{i}, x$ respectively. 
Since there is not any exact function for equation 6 , a presumed model is considered to estimate covariance function. This model must have reasonable properties and also reoresents statistical behaviors of the field variable. For instance, if the distance between $x_{i}$ and $x_{j}$ increases, this dependency should decrease. Therefore the covariance functions must be bell shaped. In this study, the following model is used:

$$
C\left(h_{i j}\right)=c_{0} e^{-\left(\frac{h_{i j}}{a}\right)^{2}}
$$

Therefore only $C_{0}$ and $a$ must be evaluated reasonably and statistically.

In this equation, the radius of domain in which correlation between values is important is quantified by parameter $a$, as is shown in Figure 1. It should be noted that the dependency in a parabolic PDE equation exists between every two points but only those points with significant dependency should be considered. These points are recognized by radius of domain using parameter $a$. In practical problems, statistical identification of this parameter is intricate. Therefore parameter $a$ should be determined base on experience.

Since in FE methods dependency among nodal values is usually outstanding in one element, parameter $a$ can be estimated by element size. Generally, $a=\sqrt{2} l_{e l}$ has yielded reasonable results.

In equation 8 , the parameter $C_{0}$ can be written as follows:

$$
c_{0}=C(0)=E\left((f-E(f))^{2}\right)
$$

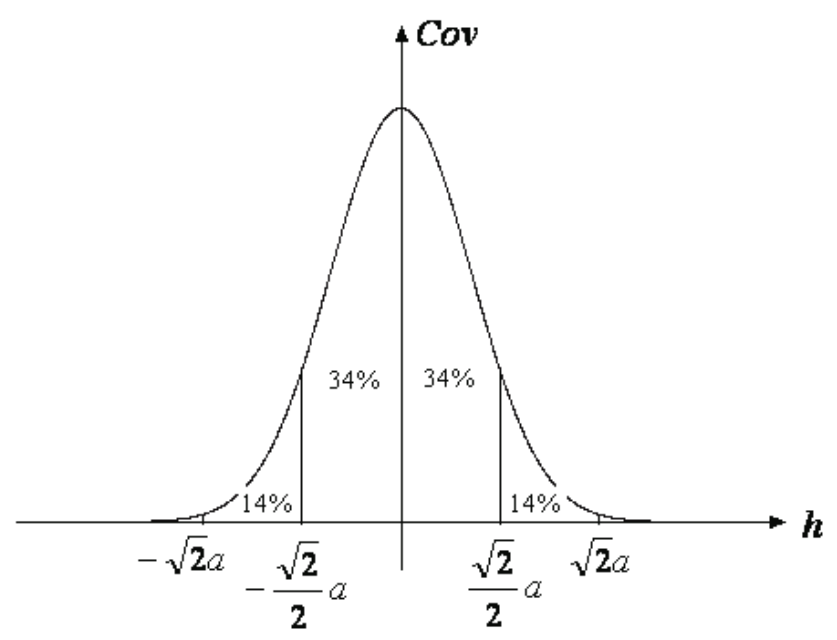

Figure 1. Gaussian model and the ratio between parameter $a$ and the area under model.
It is supposed that in a small domain, the variation of $C_{0}$ is negligible. Therefore $E\left((f-E(f))^{2}\right)$ can be evaluated statistically at every element by Arithmetic Mean and adjacent nodal values as follows:

$$
c_{0}^{I}=\sum_{i=1}^{n} \frac{\left(\hat{f}_{i}-E_{i}^{I}(f)\right)^{2}}{n}
$$

where, $C_{0}^{I}$ is calculated in element $I$. Values of $f_{i}$ is are FEM results at nodal points which were defined as the realization of field variable and $n$ is the total number of nodes in the vicinity of element $I$. The term of $E_{i}^{I}(f)$ is the mathematical expectation of field variable in relation to element $I$ at node $i$. In this paper, $E_{i}^{I}(f)$ is calculated by least square approximation in which the order of base function is similar to order of element shape function. The values of $E_{i}^{I}(f)$ for element $I$ are shown in Figure 2 schematically.

The estimation of $E_{i}^{I}(f)$ is based on two assumptions. Firstly in FE method, it is expected that the element size and the order of element shape function can characterize the field variable. Secondly, it is expected that the FEM results represent global behavior of field variable.

By using equation 8 , equation 7 can be modified as follows:

$$
\begin{aligned}
\sigma_{E}^{2}= & E\left(\left(f-f_{h}\right)^{2}\right)=c_{0}+\sum_{i=1}^{n} \sum_{j=1}^{n} \varphi_{i} \varphi_{j} c_{0} e^{-\left(\frac{\left\|X_{i}-X_{j}\right\|}{a}\right)^{2}}- \\
& 2 \sum_{i=1}^{n} \varphi_{i} c_{0} e^{-\left(\frac{\left\|X-X_{i}\right\|}{a}\right)^{2}}
\end{aligned}
$$

in which, $\sigma_{\mathrm{E}}^{2}$ is calculated at $X$ and $X_{i}$ is are nodal positions. Therefore the interpolation quality is quantified by the integration of $\sigma_{E}^{2}$ over the whole domain. Therefore:

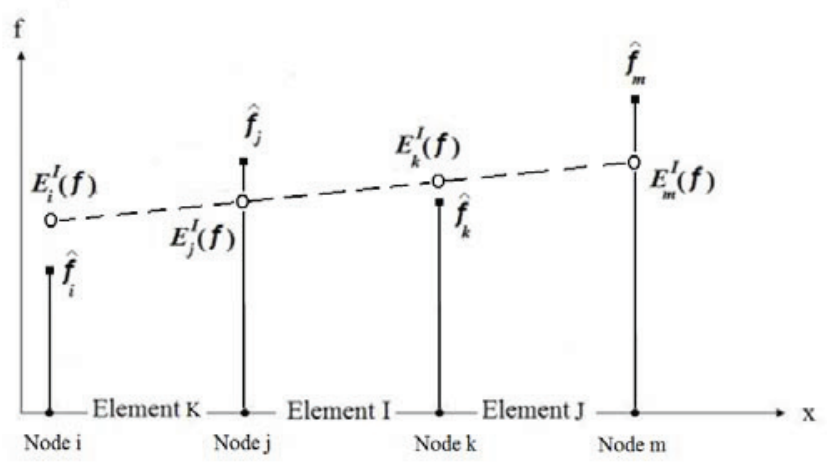

Figure 2. The estimation of $E_{i}^{I}(f)$. 


$$
\int_{\Omega} \sigma_{E}^{2} d \Omega=\sum_{e=1}^{N} \int_{\Omega_{e}} \sigma_{E}^{2} d \Omega \approx \sum_{e=1}^{N} \sum_{g=1}^{M} W_{g} \sigma_{E}^{2}
$$

where, $e$ and $g$ subscripts show the element number and the integration point number respectively in the element e. $\sigma_{\mathrm{E}}^{2}$ is calculated at integration points and $w_{g}$ is are integration weights. If equation 12 is minimized with respect to nodal positions, the best mesh is obtained. Therefore:

$$
\frac{\partial \sum_{e=1}^{N} \sum_{g=1}^{M} W_{g} \sigma_{E}^{2}}{\partial X_{i}}=\sum_{e=1}^{N} \sum_{g=1}^{M} W_{g} \frac{\partial \sigma_{E}^{2}}{\partial X_{i}}=0
$$

Equation 13 can be solved by the Newton-Raphson method. In the finite element method, shape functions are constructed by natural coordinates. Therefore shape functions are not dependent on the nodal positions. In a 2-D space, the position of $x_{k}$ is modified as follows:

$$
\left(\begin{array}{c}
\Delta x_{k} \\
\Delta y_{k}
\end{array}\right)=\psi_{1}^{-1} \psi_{0}
$$

in which, $\Psi_{0}$ is residual of equation 13 and $\Psi_{1}$ is the derivative of $\Psi_{0}$ and are calculated by nodal position of previous mesh arrangement. It can be shown that:

$$
\begin{aligned}
\psi_{1} & =-\sum_{e=1}^{N} \sum_{g=1}^{M} \frac{4 c_{0} \varphi_{k} e^{-\left(\frac{h}{a}\right)^{2}}}{a^{2}} \\
& {\left[\begin{array}{cc}
1-\frac{2\left(x_{k}-x_{g}\right)^{2}}{a^{2}} & -2 \frac{\left(x_{k}-x_{g}\right)\left(y_{k}-y_{g}\right)}{a^{2}} \\
-2 \frac{\left(x_{k}-x_{g}\right)\left(y_{k}-y_{g}\right)}{a^{2}} & 1-\frac{2\left(y_{k}-y_{g}\right)^{2}}{a^{2}}
\end{array}\right] }
\end{aligned}
$$

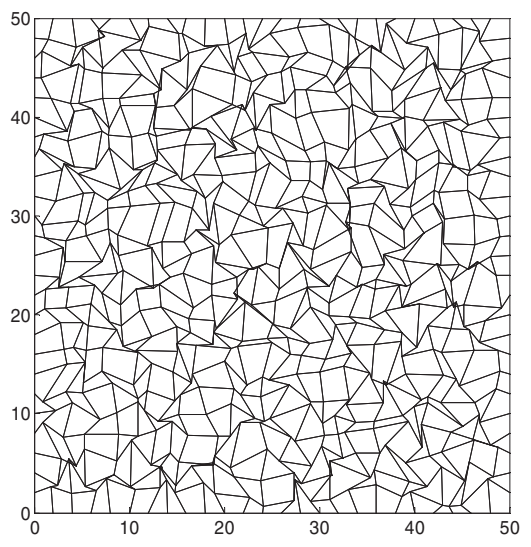

Figure 3. A randomly distorted mesh. and:

$$
\psi_{0}=-\sum_{e=1}^{N} \sum_{g=1}^{M} \frac{4 c_{0} \varphi_{k} e^{-\left(\frac{h}{a}\right)^{2}}}{a^{2}}\left[\begin{array}{l}
x_{k}-x_{g} \\
y_{k}-y_{g}
\end{array}\right]
$$

$$
b=\sqrt{\left(x_{k}-x_{g}\right)^{2}+\left(y_{k}-y_{g}\right)^{2}}
$$

where, $x_{g}$ is are the integration points. According to equations $15-\mathrm{a}$ and $15-\mathrm{b}$, the presented mesh smoothing procedure depends on the shape functions, the nodes positions, the volume of adjacent elements and the concentration of the field (identified by $C_{0}$ ) which can be calculated statistically in the relevant applications if required.

\section{Results}

In the first example of this section, capability of the presented method to reconstruct various meshes is examined. Figure 3, shows a mesh which is randomly and highly distorted. The modified meshes shown in Figures 4 and 5 have been obtained by one and five iteration of mesh smoothing. These regular meshes have been obtained when $C_{0}$ and $a$ are considered as constant parameters. The value of $C_{0}$ has no effect on the procedure but the value of $a$ must be bigger than the minimum element size. If $a$ is changed by $a=(x-10)^{2}+(y-10)^{2}+5$, the mesh is concentrated at point $(10,10)$ as is shown in Figure 6. Also if the definition of $\frac{h_{i j}}{a}$ in equation 8 is replaced by:

$$
g_{x x} \cdot \Delta x^{2}+g_{y y} \cdot \Delta y^{2}+g_{x y} . \Delta x \cdot \Delta y
$$

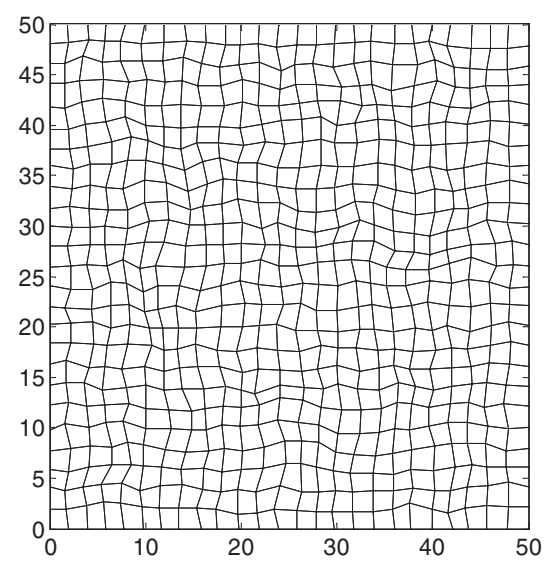

Figure 4. Reconstructed mesh obtained from the mesh in Figure 3. by one iteration of mesh smoothing. Here, $C_{0}$ and $a$ are constants. 
mesh concentration will be anisotropic. For example if in equation $16 g_{x x}=1 / a_{x}^{2}, g_{y y}=1 / a_{y}^{2}$ and $g_{x y}=0$, in Figure 7 anisotropic concentration is obtained by following covariance function:

$C\left(h_{i j}\right)=c_{0} e^{\left.-\left[\frac{x_{i j}}{a_{x}}\right)^{2}+\left(\frac{y_{i j}}{a_{y}}\right)^{2}\right]}, a_{x}=(x-10)^{2}+(y-10)^{2}+5, a_{y}=2$

In the above examples, the presented method has been fairly stable and rapidly converged. In this stage, the time cost of this method is in the order of volumetric approach.

The presented method can be applied for a complicated geometry. Figures 8 and 10 show initial meshes. Smoothed meshes are shown in Figures 9 and 11 respectively. As it can be seen the mesh is affected by the geometry of the domain. In these examples, since $C_{0}$ and $a$ are considered to be constant, the resultant smoothed meshes reflect the shapes of the boundaries.

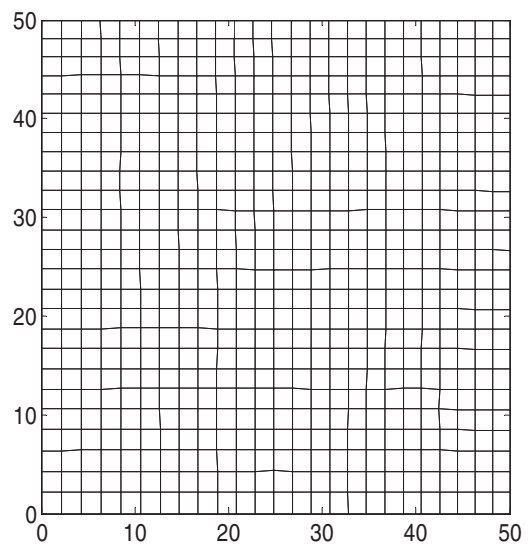

Figure 5. Reconstructed mesh obtained from the mesh in Figure 3 by 5 iteration of mesh smoothing. Here, $C_{0}$ and $a$ are constants.

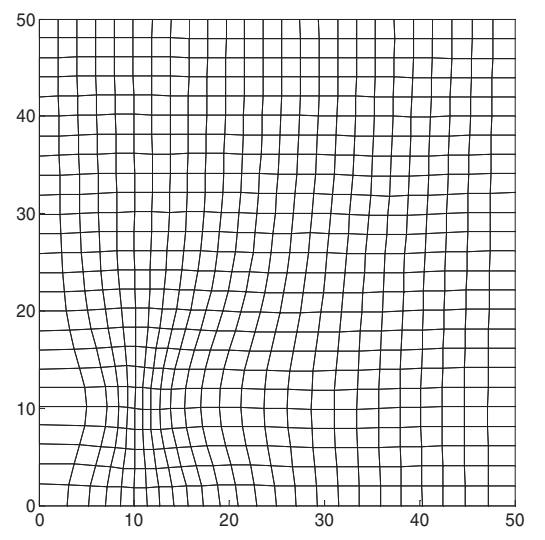

Figure 7. Reconstructed mesh by eq. 17 .
If a primary solution of field exists or an explicit FEM procedure is used, $C_{0}$ can be calculated statistically. Figure 12 shows this parameter for a quadrant rectangular elastic plate with a circular hole under static in plain stress loading. Based on statistical values of $C_{0}$, the variance of interpolation is calculated and plotted in Figure 13. The improved mesh is reconstructed in Figures 14 and 15 by using 5 iterations and 30 iterations respectively. Also Figure 16 shows that the error of stress field on y-axis obtained by the presented adaptive mesh has reduced by further iterations. This figure also shows that this adaptive approach is quite stable and error values do not increase by increasing iterations. In this study, the same procedure is used to reconstruct the adaptive mesh for a thermal analysis. In this example, a plate is traversed by a moving heat flux and the consequence adaptive mesh is computed by presented approach. Since the statistical values of $C_{0}$ in

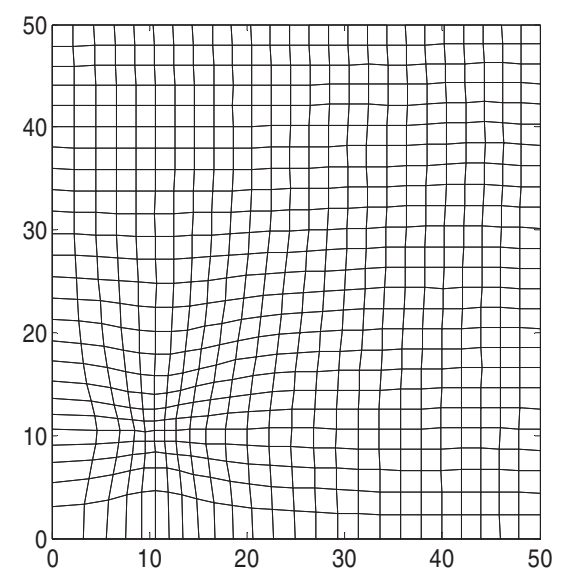

Figure 6. Reconstructed mesh, $C_{0}$ is constant and $a=(\mathrm{x}-10)^{2}$ $+(\mathrm{y}-10)^{2}+5$.

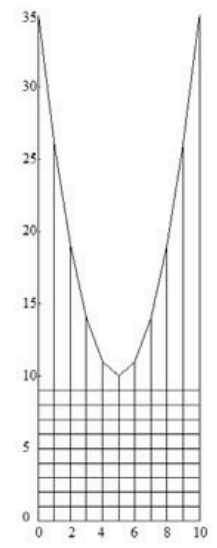

Figure 8. Initial mesh. 


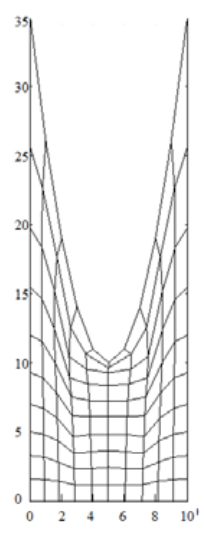

Figure 9. Reconstructed mesh.

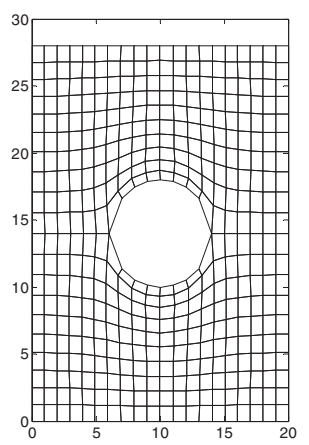

Figure 11. Reconstructed mesh.

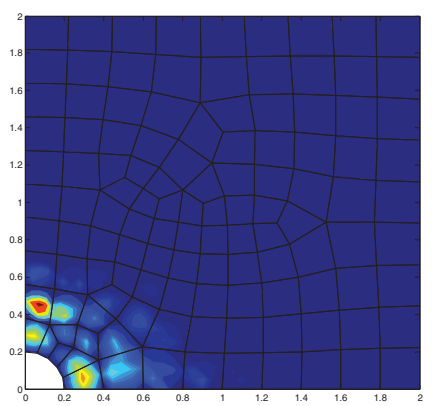

Figure 13. Contour of $\sigma_{\mathrm{E}}^{2}$.

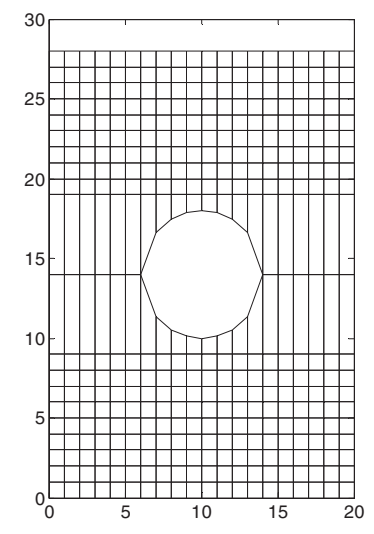

Figure 10. Initial mesh.

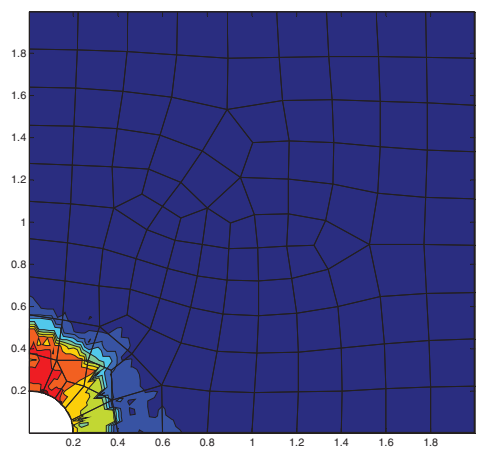

Figure 12. Contour of $C_{0}$.

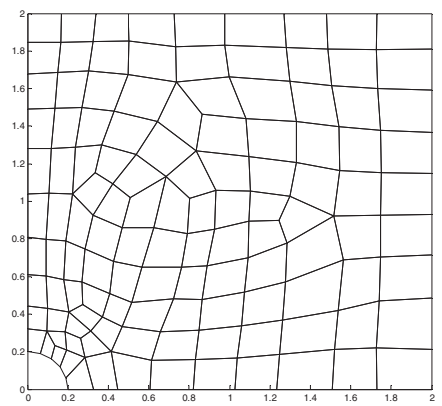

Figure 14. The reconstructed mesh by 5 iteration of mesh smoothing.

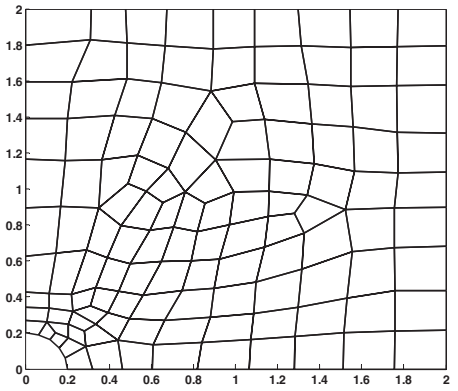

Figure 15. The reconstructed mesh by 40 iteration of mesh smoothing. 
some elements are zero, computational problems occur. Therefore in the elements in which statistical value of $C_{0}$ is less than 20 percent of mean value of $C_{0}$, its mean value is considered.

Figures 17-20 show the adaptive constructed meshes and the temperature fields in the thermal model of line heating analysis. In this example, value of $C_{0}$ is considered as a function of heat flux distribution. To show the effect of mesh adaptively on the results, temperature values are examined. The maximum temperature value in the last increment is $808^{\circ} \mathrm{C}$. In this example the temperature values in the last increment are also obtained without mesh reconstruction, by the same element size (Figure 21) as well as a finer element size (Figure 22). The maximum temperature values are $760^{\circ} \mathrm{C}$ and $808.5^{\circ} \mathrm{C}$ respectively. As it can be seen the error is reduced from $7 \%$ down to less than $1.4 \%$ by mesh reconstruction. In an ordinary

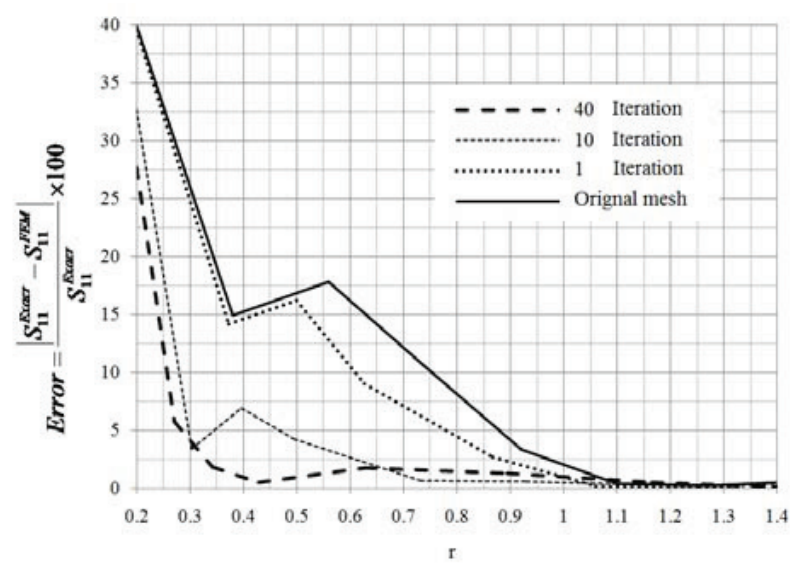

Figure 16. The error of stress field on y-axis.

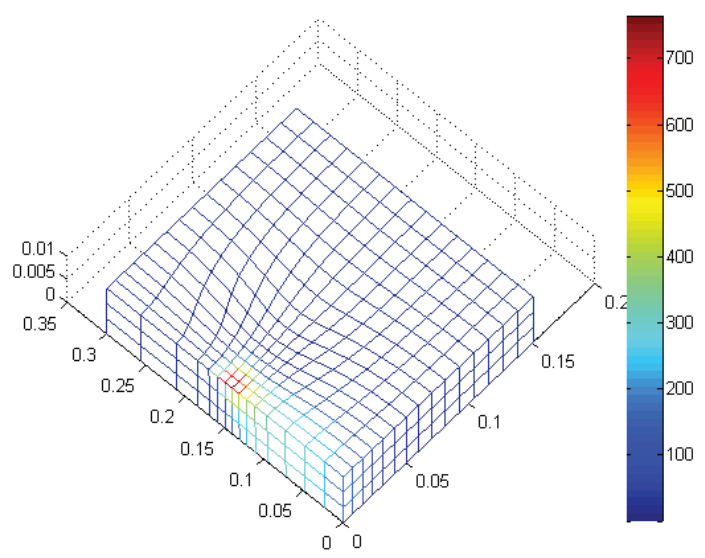

Figure 18. The contour of temperature and reconstructed mesh at $\mathrm{t}=10$. laptop (2.00 GHz) CPU time without smoothing takes 180 seconds and with smoothing takes 222 seconds and 241 seconds when temperature updates are is also performed. Hence smoothing time cost is about $22 \%$ of the total cost and $33 \%$ if updating of field variable is also performed. It is been noted that CPU time without smoothing takes 1026 seconds for finer element size.

\section{Conclusion}

In this paper, a probabilistic adaptive mesh smoothing method was introduced. The affects of mesh characteristics and also variation of a primary field (if available) on the accuracy of results were considered directly in the presented approach.

The capability of the presented method was examined by various examples. Firstly, by several examples the ability

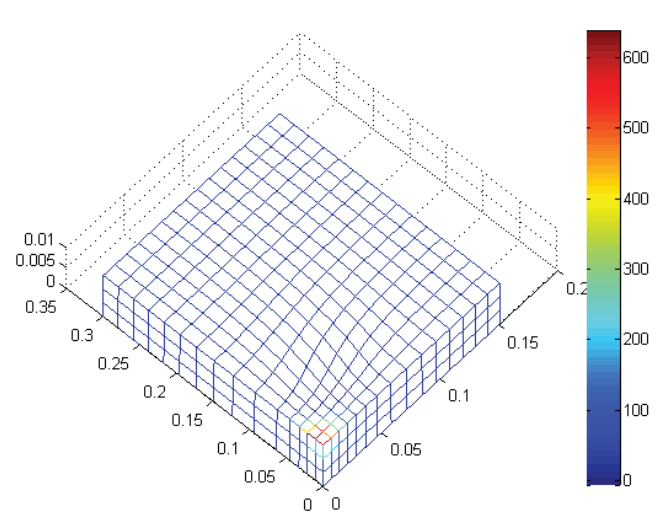

Figure 17. Temperature contour and reconstructed mesh at $\mathrm{t}=0$.

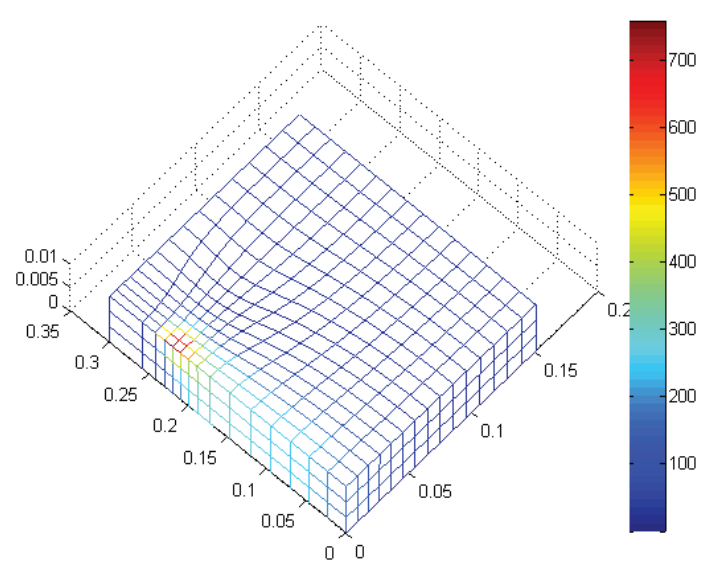

Figure 19. The contour of temperature and reconstructed mesh at $\mathrm{t}=15$. 


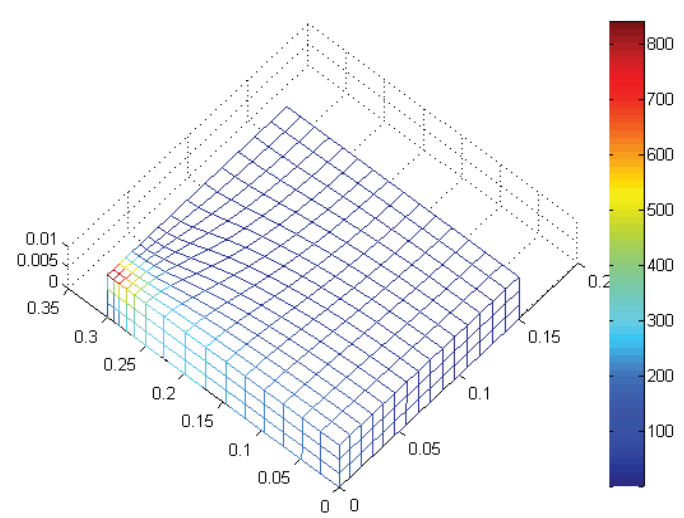

Figure 20. The contour of temperature and reconstructed mesh at $\mathrm{t}=20$.

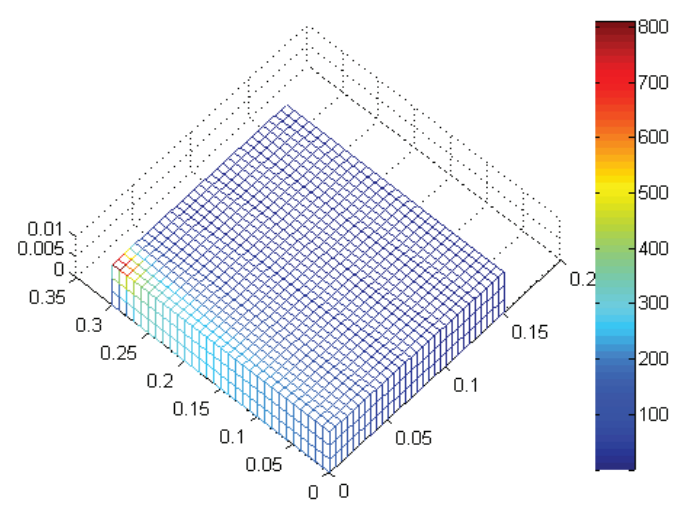

Figure 22. The contour of temperature at $t=20$, unchanged fine mesh.

of the method to reconstruct various mesh geometries such as uniform mesh, isotropic concentrated mesh and also anisotropic concentrated mesh were demonstrated. Secondly, mesh reconstructions of domains with curved boundaries were performed through two examples. Then the steps of mesh smoothing approach were shown for a rectangular plate with a circular hole under in plain loading. Finally presented method was used for an ALE example. To the authors' knowledge there is no available adaptive mesh smoothing method which directly considers field variation. In other words available techniques are either adaptive refinement which consider field variable such as Z-Z method or are smoothing methods such as Laplacian or volumetric etc which may be used sequentially but not simultaneously.

It was shown that, the presented mesh smoothing procedure considers nodes positions and volume of adjacent elements. Intensity of the field which is identified by $C_{0}$ and $a$ parameters could be calculated statistically in the relevant applications.

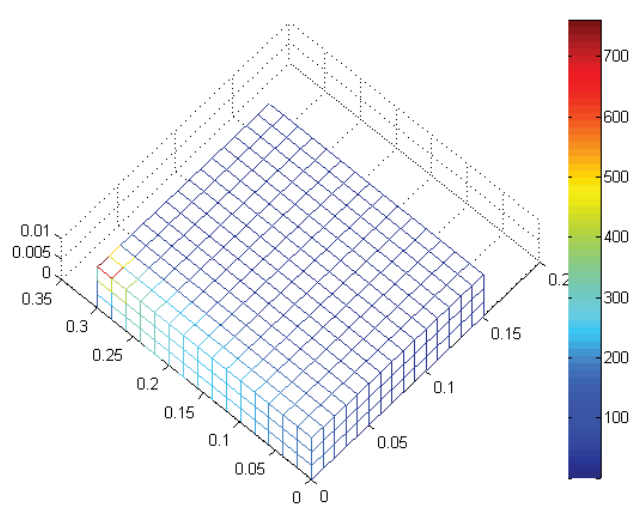

Figure 21. The contour of temperature at $t=20$, unchanged coarse mesh.

\section{References}

1. Freitag LA. On combining Laplacian and optimizationbased mesh smoothing techniques. AMD Trends in Unstructured Mesh Generation, ASME. 1997; 220:37-43.

2. Ali-Yahia DA, Baruzzi G, Habashi WG, Fortin M, Dompierre J, Vallet MG. Anisotropic mesh adaptation: towards user-independent, mesh-independent and solverindependent CFD, Part II: structured grids. International Journal for Numerical Methods for Heat and Fluid Flow. 2002 Jul; 39(8):657-73.

3. Tam A, Ali-Yahia DA, Robichaud MP, Moore M, Kozel V, Habashi WG. Anisotropic mesh adaptation for 3D flows on structured and unstructured grids. Comput Method Appl M. 2000; 189:1205-30.

4. Bossen F. Anisotropic mesh generation with particles. [Master's Thesis]. [Pittsburgh, PA]: Carnegie Mellon University, cMU-CS-96-134; 1996 May.

5. Thompson JF, Warsi ZUA, Mastin CW. Numerical grid generation, foundations and applications. NewYork: NorthHolland; 1985.

6. Eiseman PR. Alternating direction adaptive grid generation, American Institute of Aeronautics and Astronautics Journal. AIAA Paper 83-1937; 1983.

7. Spekreijse SP. Elliptic grid generation based on Laplace equations and algebraic transformations. J Comput Phys. 1995; 118(1):38-61.

8. Soni BK, Koomullil R, Thompson DS, Thornburg H. Solution adaptive grid strategies based on point redistribution. Comput Method Appl M. 2000; 189:1183-1204.

9. Canann S, Stephenson M, Blacker T. Optismoothing: an optimization driven approach to mesh smoothing. Finite Elements Anal Design. 1993; 13(2-3):185-190.

10. Siroisa Y, Dompierreb J, Valletc MG, Guibaultc F. Hybrid mesh smoothing based on Riemannian metric nonconformity minimization. Finite Elements Anal Design. 2010; 46(1-2):47-60. 
11. Xu H, Newman TS. An angle-based optimization approach for 2D finite element mesh smoothing. Finite Elements Anal Design. 2006; 42(13):1150-64.

12. Freitag LA, Gooch CO. Tetrahedral mesh improvement using swapping and smoothing. Int J Numer Meth Eng. 1997; 40:3979-4002.

13. Freitag L. On combining Laplacian and optimization-based mesh smoothing techniques. Proceedings of the Sixth International Meshing Roundtable, AMD. 1997; London. 220:375-90.

14. Canann SA, Tristano JR, Staten ML. An approach to combined Laplacian and optimization-based smoothing for triangular, quadrilateral, and quad-dominant meshes. Proceedings of the Seventh International Meshing Roundtable; 1998; Dearborn, MI. p. 479-94.

15. Babuska I, Rheinboldt WC. A posteriori error estimates for the finite element method. Internat. J Numer Methods Eng. 1978; 12:1597-1615.

16. Babuska I, Strouboulis T. The finite element method and its reliability. Oxford: Clarendon Press; 2001.

17. Zienkiewicz OC, Zhu JZ. A simple error estimator and adaptive procedure for practical engineering analysis. Int. J Numer Methods Eng. 1987; 24:337-57.
18. Zienkiewicz OC, Zhu JZ. The Superconvergent Patch Recovery (SPR) and adaptive finite element refinement. Comput. Methods Appl Mech Eng. 1992; 101:207-24.

19. Zienkiewicz OC, Zhu JZ. The superconvergent patch recovery and a posteriori error estimates. I: The recovery technique. Int J Numer Methods Eng. 1992; 33:1331-64.

20. Zienkiewicz OC, Zhu JZ. The superconvergent patch recovery and a posteriori error estimates. II: Error estimates and adaptivity. Int J Numer Methods Eng. 1992; 33: 1365-82.

21. Boroomand B, Zienkiewicz OC. Recovery by Equilibriumin Patches (REP). Int J Num Meth Eng. 1997; 40:137-54.

22. Boroomand B, Zienkiewicz OC. An improved REP recovery and the effectivity robustness test. Int J Num Meth Eng. 1997; 40:3247-77.

23. Zhang Z, Naga A. A New Finite element gradient recovery method: superconvergence property. SIAM Journal on Scientific Computing. 2005; 26(4):1192-213.

24. Matheron G. Traite de Geostatistique Appliquee, Tome 1. Memoires du Bureau de Recherches Geologiques et Minieres, No. 14. Editions Technip, Paris; 1962.

25. DeFinetti B. Decisions and Proper Scoring Rules In: Mura A, editor. Philosophical lectures on probability. Springer Science, Business Media B.V; 2008. 